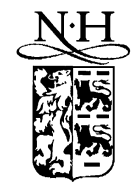

ELSEVIER

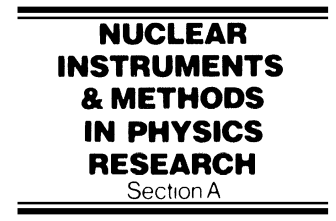

\title{
A study of breakdown limits in microstrip gas counters with preamplification structures
}

\author{
P. Fonte ${ }^{\mathrm{a}}$, V. Peskov ${ }^{\mathrm{b}}$, B.D. Ramsey ${ }^{\mathrm{b}, *}$ \\ ${ }^{a}$ LIP/Coimbra University and Instituto Superior de Engenaria de Coimbra, Portugal \\ ${ }^{\mathrm{b}}$ NASA, Marshall Space Flight Center, Huntsville, Alabama 35812, USA
}

Received 3 November 1997; received in revised form 15 February 1998; accepted 30 March 1998

\begin{abstract}
We have studied the charge and breakdown limits of Microstrip Gas Counters (MSGC's) with two different preamplification structures: the Gas Electron Multiplier (GEM) and the Parallel-Plate Avalanche Counter (PPAC). It was found that in both cases the breakdown limit was increased by 1-2 orders of magnitude compared to the bare MSGC and that this was due to the spreading of the primary electron cloud during pre-amplification. This spreading reduces the charge density in the final MSGC avalanche, permitting much higher total gains before streamers form. The real practical gain limitations in these two-stage detectors arose not from sparking, but from a loss of proportionality due to space charge effects. (C) 1998 Elsevier Science B.V. All rights reserved.
\end{abstract}

\section{Introduction}

As was shown in our previous papers [1-3], gain-limiting breakdown in MSGCs is caused by surface streamers, which, because of their attachment to the substrate, are unquenched and once started transit rapidly to sparks. This limits the maximum achievable gains in conventionally designed MSGCs to about $10^{4}$. It was thus concluded that the substrate represented a weak point and that in optimized designs, in which the role of substrate was reduced, one could achieve much higher gains [2].

A different approach to increasing the MSGC gain was proposed in Ref. [4], where a new pre-

\footnotetext{
*Corresponding author. Tel.: + 1256544 7743; fax: 1256 544 7754; e-mail: brain.ramsey@msfc.nasa.gov.
}

amplification structure - The Gas Electron Multiplier (GEM) - was suggested. The combination of GEM and the MSGC (GEM + MSGC), where the GEM is used to preamplify the charge signal before it enters the MSGC, permitted much higher total avalanche charges before sparking appeared [5].

We have studied the achievable gain limits in MSGCs combined with the GEM. We present our findings, and as a direct result of these encouraging measurements, we have also investigated an alternative approach using a Parallel-Plate Avalanche Counter (PPAC) as a preamplification front end for the MSGC.

\section{Experimental set up}

Our experimental set up is shown schematically in Fig. 1 and consists of a test chamber inside of which 


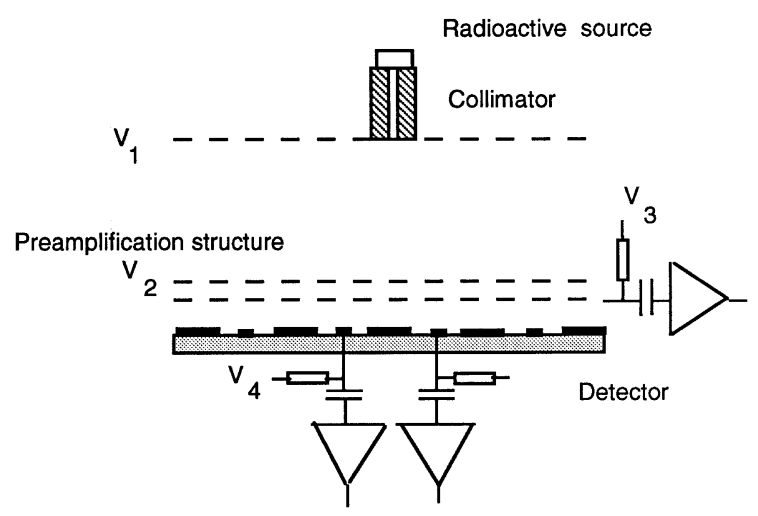

Fig. 1. Schematic drawing of the test chamber.

was installed a "detector" and, directly above it, a "preamplification structure". As preamplification structures both the GEM and a PPAC were tested. The GEM was obtained from CERN, Switzerland, and had a $10 \times 10 \mathrm{~cm}^{2}$ active area. The specifics of its geometry are described in detail in Ref. [5]. The PPACs were assembled from two round frames with inner diameters of $5 \mathrm{~cm}$. On the top of each frame a metallic mesh, of $90 \%$ optical transparency, was stretched and glued. Two PPACs were tested: one with a $3 \mathrm{~mm}$ gap between the meshes and the other with a $1.5 \mathrm{~mm}$ gap.

As detectors, we tested not only MSGCs, but also a Multiwire Proportional Counter (MWPC), and the PPACs described above. Two different MSGCs were used, the first (MSGC\#1) having a straight $1 \mathrm{~mm}$ anode pitch over its whole active area and the other (MSGC \#2) having a split pattern, one half with $200 \mu \mathrm{m}$ pitch and the other with a $400 \mu \mathrm{m}$ pitch. The $1 \mathrm{~mm}$ pitch unit had $20 \mu \mathrm{m}$ wide anodes and was fabricated by IMT, Switzerland, to a design described in Ref. [6]. The $200 / 400 \mu \mathrm{m}$ pitch unit had $10 \mu \mathrm{m}$ wide anodes and was manufactured by Max Levi, USA, to a design described in Ref. [7]. In some MSGC measurements neighboring anode strips, that were normally all connected, were separated from the group and connected to individual preamplifiers. The MWPC detector was made from a plane of $20 \mu \mathrm{m}$ diameter anode wires, on a $2 \mathrm{~mm}$ pitch, sandwiched between two orthogonal planes of $0.7 \mathrm{~mm}$-diameter cathode wires, also on a $2 \mathrm{~mm}$ pitch. Its active area was $2 \times 3 \mathrm{~cm}^{2}$.
The distances between the preamplification structure and the detector was between 4 and $10 \mathrm{~mm}$, depending on the particular measurement. The drift region, above the preamplification structure, was variable between 1 and $5 \mathrm{~cm}$. Measurements were performed in various Ar and Xe-based mixtures at pressures from 0.1 to $1 \mathrm{~atm}$. As in Ref. [1], $\mathrm{Fe}^{55}(6 \mathrm{keV}), \mathrm{Cd}^{109}(22 \mathrm{keV})$, an $\mathrm{X}$-ray gun (6 $\mathrm{keV}$ photons) and alphas were used to produce primary ionization. To enable visual recording of the streamers, the test chamber was equipped with a transparent window (see details in Ref. [2].)

\section{Results}

\section{1. $G E M+M S G C$ and $G E M+M W P C$}

Our measurements with the GEM preamplification structure coupled to both microstrip detectors and multiwire counters, GEM + MSGC and GEM + MWPC, confirmed the results obtained in Ref. [5]. As an example, Fig. 2 shows the total gain on the MSGC vs. the voltages applied to the MSGC and GEM, measured with $\mathrm{Fe}^{55}$. One can clearly see that when preamplification is used, the total overall gain (or total avalanche charge) in the MSGC, before breakdown, increases dramatically. Similar results were obtained with the MWPC. At high GEM gains, the overall system gains were so large that space charge effects in the MSGC avalanche were the performance-limiting factor. These results, the total gain increase and the onset of space charge effects, were confirmed in various $\mathrm{Ar}$ and Xe-based mixtures at pressures from 0.1 to $1.5 \mathrm{~atm}$. In Ar-based mixtures, at low pressures, the space charge effect was, however, much less pronounced. Typical energy resolutions with GEM were around $19-20 \%$ FWHM at $6 \mathrm{keV}$ at very low gas gains $(<5)$ degrading to around $22-25 \%$ FWHM at higher gains.

While in full agreement with others, the overall gain results are somewhat surprising. It is well known [3] that the transition of avalanches to breakdown-forming streamers depends mostly on the total charge in the avalanche or, to be more precise, on the charge density. A large increase in total charge, must therefore be accompanied by 


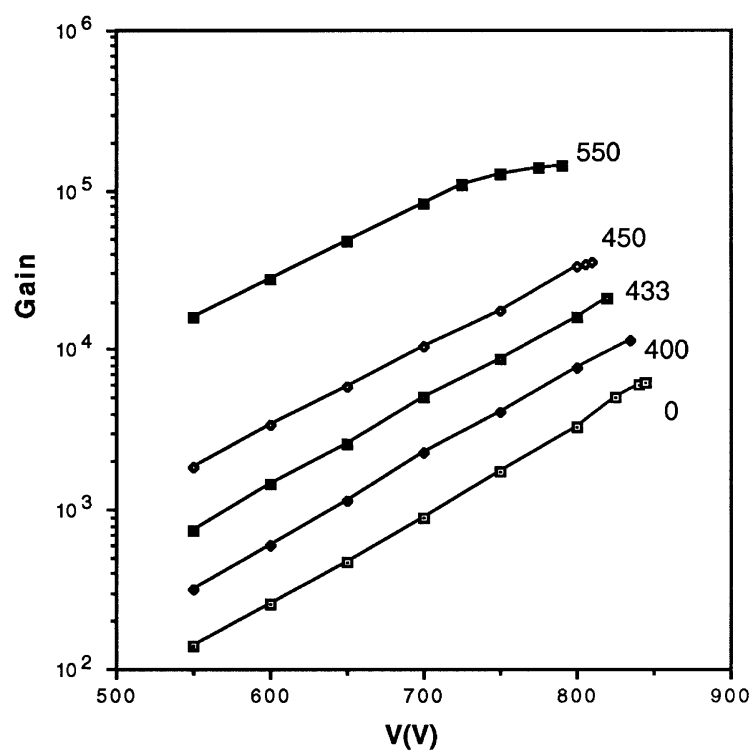

Fig. 2. Total gain vs. voltages for MSGC \#1+ the GEM preamplification structure measured with $\mathrm{Fe}^{55}$. The gas mixture was $\mathrm{Ar}+\mathrm{CH}_{4}(5 \%)$ at $1.05 \mathrm{~atm}$. The numbers near the curves correspond to the voltages applied to GEM. The distance between the GEM and the MSGC was $10 \mathrm{~mm}$.

a correspondingly large decrease in total charge density to avoid breakdown conditions.

\subsubsection{The effects of preamplification on the charge cloud size}

In an attempt to understand the resulting charge densities under preamplification conditions, we performed calculations of diffusion for a GEM-like multiplication geometry (see Fig. 3 and the Appendix). These calculations indicated that the primary electron charge cloud may experience a strong diffusion in the GEM structure, resulting in a much lower charge density for the preamplified signal.

As an independent check, we also performed measurements of diffusion of the primary electrons when undergoing preamplification. In these measurements, the X-ray (iron-K) beam from a generator was collimated with a slit $6 \mathrm{~cm}$ long and $100 \mu \mathrm{m}$ wide. To minimize divergence of the beam, and diffusion of the charge cloud before preamplification, the drift and absorption gap was kept at a practical minimum, in this case $10 \mathrm{~mm}$. For similar reasons, the transfer gap, between the
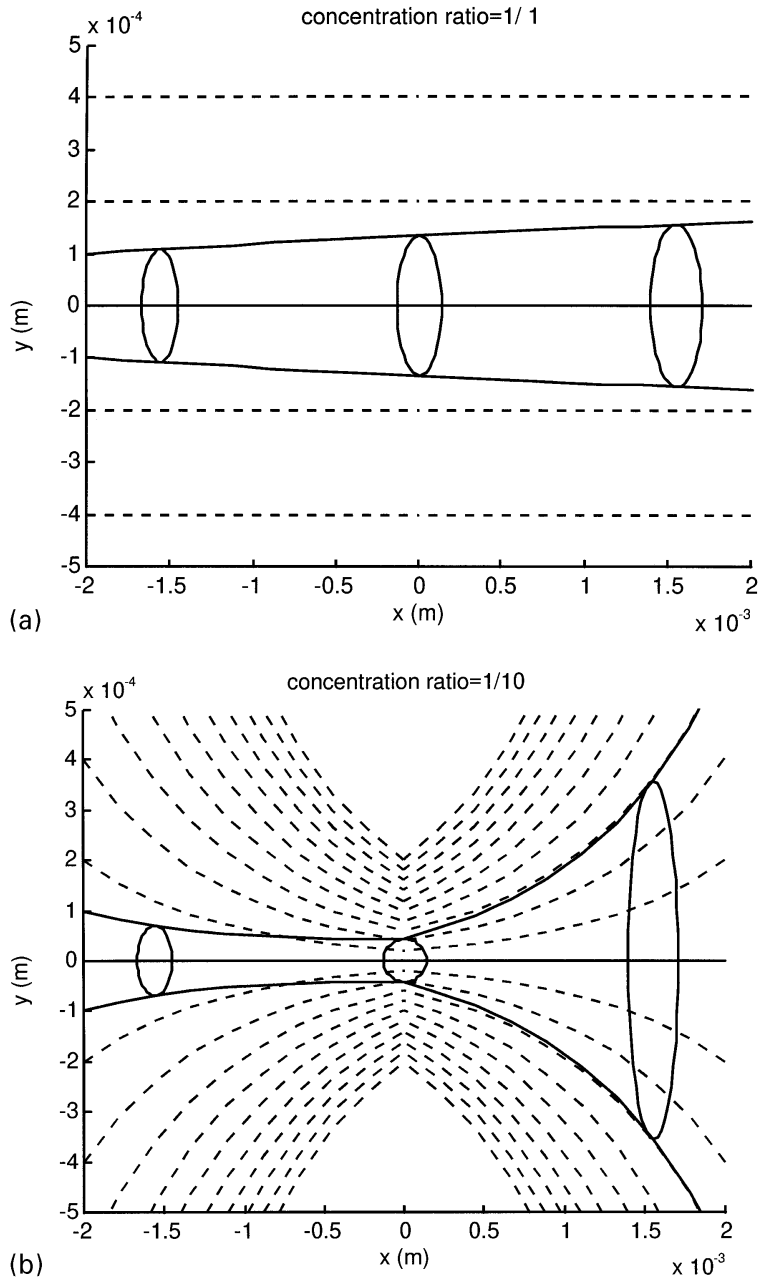

Fig. 3. Calculated evolution of the electron cloud for a constant field (a) and for a GEM-like focusing-defocusing field (b). The focusing factor is 10 (see the appendix for more details.) The dashed lines represent field lines. The solid line at $y=0$ defines the center of the drifting electron cloud. The other solid lines denote the boundary of the diffused cloud.

preamplification structure and the detector was kept to just $4 \mathrm{~mm}$ for this set of measurements. With this geometry, the effective X-ray-beam width was estimated to be less than $200 \mu \mathrm{m}$.

Three sets of measurements were performed. In the first set, the signal was taken from a single anode strip and the collimated (Fe K) X-ray beam was scanned normal to the anode. In these measurements we attempted to align the GEM's 
holes parallel to, and centered on the microstrip anodes. To register the events, the MSGC with the $200 / 400 \mu \mathrm{m}$ pitch was used. When the X-ray beam

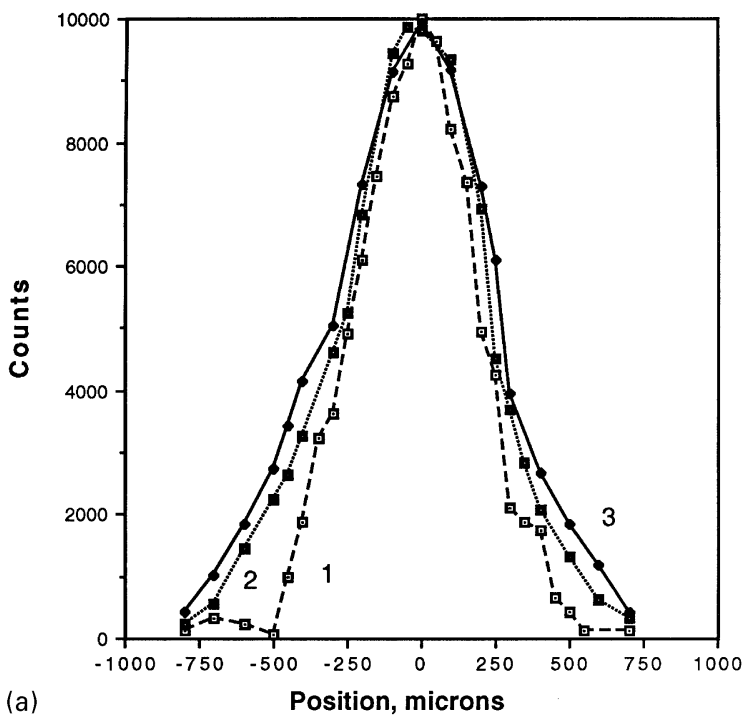

illuminated a particular strip, the pulse-height spectra from this strip contained the rather broad main line at $6.4 \mathrm{keV}$ and the argon escape peak at
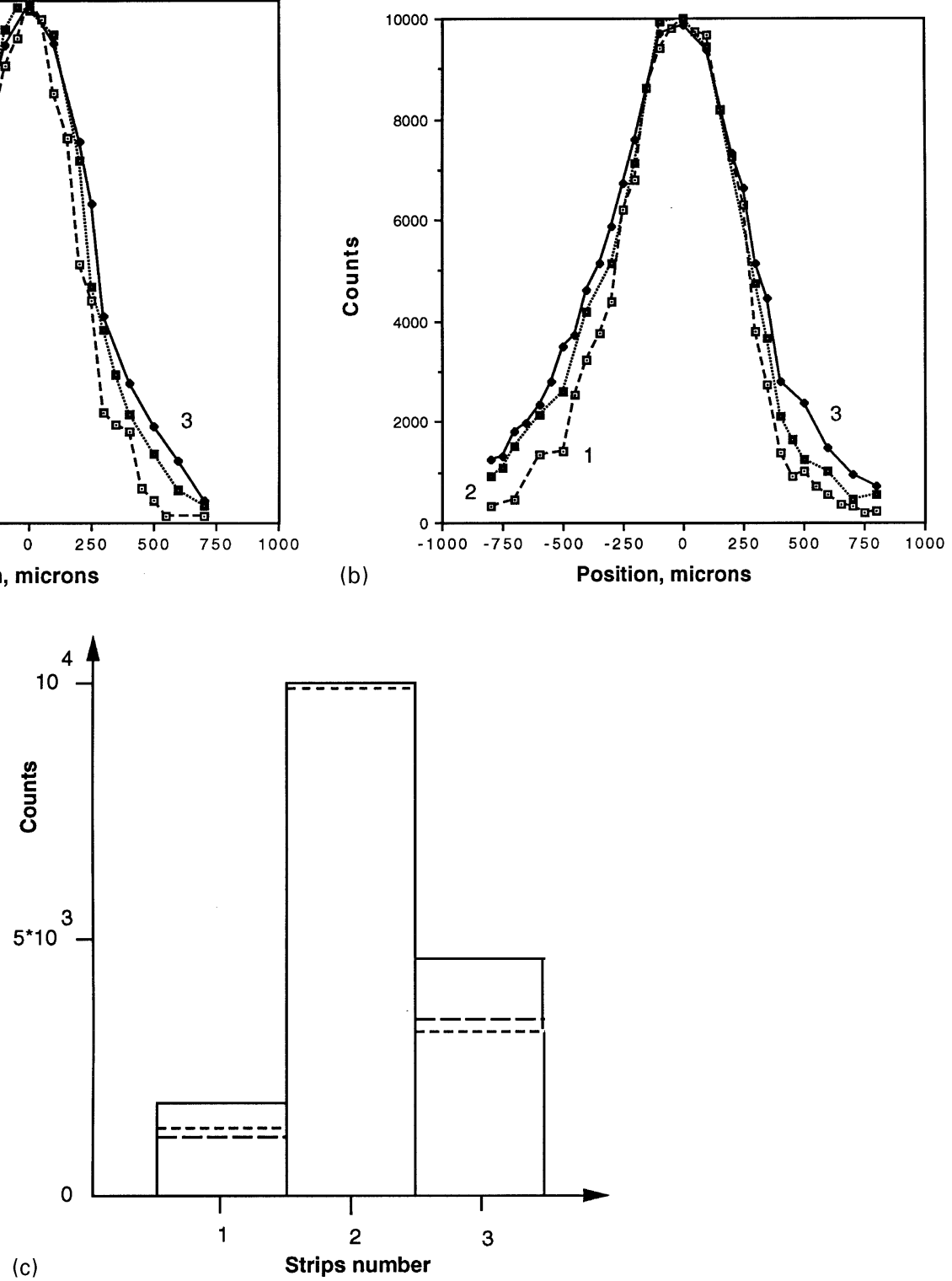

Fig. 4. (a) Number of counts as a function of the beam position for a threshold $15 \%$ below the maximum peak height (1). Curves (2) and (3) correspond to the cases where the GEM and the PPAC were used as preamplification structures, both at effective gains of 50. Measurements were done in P10 gas at 1.05 atm. The MSGC \# 2 with a $200 \mu \mathrm{m}$ pitch was used and the gap between the GEM/PPAC and the MSGC was $4 \mathrm{~mm}$. (b) The same as in Fig. 4(a), but for a threshold of $25 \%$ below the maximum anode peak height. (c) Number of counts for three neighboring strips for a fixed collimated beam position using the $400 \mu \mathrm{m}$ section of MSGC \# 2 with the GEM positioned $4 \mathrm{~mm}$ above it. The gas mixture was again P10 at $1.05 \mathrm{~atm}$. The dashed lines represent two independent runs without the GEM. The solid line, with the GEM preamplification. 
$3.4 \mathrm{keV}$. When the beam was moved some distance away to neighboring regions the pulse-height spectra from the strips contained only the argon fluorescent lines at $3 \mathrm{keV}$. To avoid measuring this fluorescent feature, scalar thresholds $15 \%$ and $25 \%$ below the maximum peak height were set and events counted as a function of beam position. Results of these measurements are presented in Fig. 4(a) and Fig. 4(b). In spite of some misalignments, one can see that after multiplication in GEM the electron cloud becomes larger, although the effects are somewhat masked by additional broadening due to the beam width and the initial charge cloud size. In the second set of measurements the position of the collimated beam was fixed and signals were taken from three neighboring strips with the scaler threshold once again adjusted $15 \%$ below the maximum signal amplitude. Fig. 4(c) shows the results of these measurements. Depicted are the number of counts from two neighboring strips for two conditions; with and without preamplification in GEM. These results are in agreement with those obtained in the first set of measurements, and show an increase in chargecloud size after preamplification, of roughly $15 \%$. This measured profile, however, is a convolution of the cloud size and the beam width, and hence corresponds to a proportionally larger increase in charge cloud size. A best estimate of this is around a factor of two.

Since the above two sets of measurements rejected many events due to the necessarily high threshold cuts, and were thus strictly speaking not true diffusion measurements, we also performed a third set using a more standard approach. Here, we measured the time duration of the current pulse, produced by $6 \mathrm{keV} \mathrm{X}$-ray photons, with and without preamplification by the GEM structure. For these measurements, the MSGC detector was replaced by a PPAC with a $3 \mathrm{~mm}$ gap (GEM + PPAC) which allowed operation at gains high enough to measure current pulses. All the measurements were done in Argon $+10 \%$ Methane at a total pressure of $1 \mathrm{~atm}$. The gap between GEM and the PPAC was held, for practical reasons at $\sim 1 \mathrm{~cm}$. The pulse durations measured were 25 and 12 ns FWHM, with and without GEM preamplification, respectively, and while it is very difficult to determine precise longitudinal extents from these data, they nevertheless indicate a substantial additional spreading of the charge cloud after multiplication in the GEM

Now, it is known that, at low gains, avalanches in MSGCs and MWPCs start to develop close to the strips or wires, typically at a distance comparable to the electrode diameters $[8,9]$. Therefore, typical avalanche sizes in both MSGCs and MWPCs, are a few tens of microns. In this case a small diffusion of the primary electron cloud, say around $100 \mu \mathrm{m}$, will cause a spreading of the avalanches comparable to their sizes and as a result the charge densities will drop noticeably. In the case of PPACs, however, the avalanche size is much greater. Our estimates, based on diffusion models $[9,10]$, predict avalanches on the order of $200-300 \mu \mathrm{m} \mathrm{rms}$ at $1 \mathrm{~atm}$, in agreement with published data [11-13]. In this case, the additional broadening due to preamplification will have a much smaller effect on the overall charge density, so that one can expect that preamplified PPACs will show little improvement in gain characteristics. To test this, measurements were performed with the GEM + PPAC combination.

\section{2. $G E M+P P A C$}

The GEM + PPAC combination was now used to explore its gain characteristics. At first, the PPAC gain was kept relatively low so that the effect of increasing the gain in the GEM structure could be carefully explored. Here, $\mathrm{Fe}^{55}$ was used as a source of ionization. Then, for a given GEM gain, the voltage on the PPAC was slowly increased until breakdown appeared. By measuring the current pulse from the PPAC just before breakdown, one can calculate the total charge deposited in the avalanche.

The results of measurements with the GEM + PPAC are presented in Fig. 5. One can see that in spite of multiplication in GEM there is no big increase in the total collected charge in the PPAC detector. This confirms qualitatively our expectations, that spreading of the charge cloud by the GEM does not noticeably affect the charge density in the final (PPAC) avalanche, and that the overall gain, therefore, is not noticeably increased. 


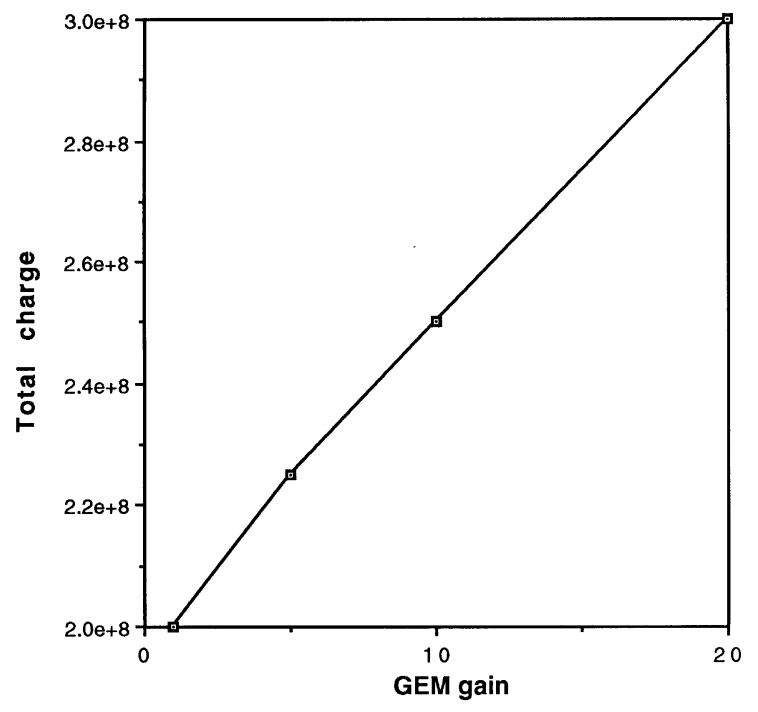

Fig. 5. Total charge in the avalanches (in electrons) at which breakdowns appear, in the $3 \mathrm{~mm}$ gap PPAC detector, as a function of GEM preamplification gain. Measurements were done with $\mathrm{Fe}^{55}$ in $\mathrm{Ar}+\mathrm{CH}_{4}(5 \%)$. The distance between the GEM and the PPAC was $\sim 1 \mathrm{~cm}$.

\section{3. $P P A C+M S G C$}

As was found with the GEM + MSGC and discussed above, a large overall charge would also be expected for the PPAC-amplified MSGC. Our measurements fully confirm this assumption. Fig. 6 shows the total charge collected on the MSGC, as a function of the voltages on the MSGC and PPAC. No MSGC breakdowns were observed during these measurements at high PPAC gains, the plotted limit being set by strong charge saturation only. Indeed, provided the MSGC voltage was kept below $760 \mathrm{~V}$ (Fig. 6), then no breakdowns would ever be observed, and any desired gain could be achieved simply by increasing the effective preamplification. Breakdowns appeared at total gains $>10^{6}$ but in the PPAC rather than the MSGC. This is because only part of the charge is extracted from the PPAC in to the MSGC, so its gain is also igh (see discussion for more details). It is known that the transparency of meshes for electron clouds is proportional to the ratio of the field on both sides. In our case, this field ratio was typically $3 \%$ and so the transparency was of the same order. The

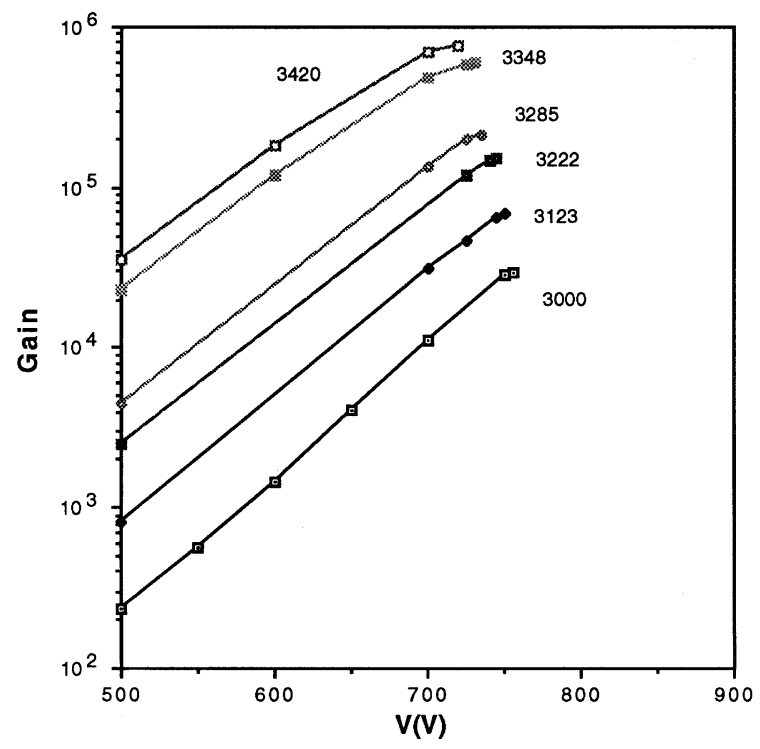

Fig. 6. Total gain vs. voltage for the PPAC (3 mm gap) + MSGC \# 1 configuration in $\mathrm{Ar}+\mathrm{CH}_{4}(5 \%)$ at $1.05 \mathrm{~atm}$. The numbers near the curves correspond to the voltages applied to the PPAC. The distance between PPAC and MSGC was $10 \mathrm{~mm}$.

design of our test chamber prevented this from being increased significantly. We were not able to reduce the gap between PPAC and MSGC to less than $4 \mathrm{~mm}$ and our $\mathrm{HV}$ feed-through could not withstand voltages more than $5 \mathrm{kV}$. This limited the available transfer field and hence the effective transfer ratio.

Comparing Figs. 2 and 6, it can be seen that the overall gain with the PPAC-preamplified MSGC is about factor of 3 higher than with GEM. A key factor may be the relatively large distance over which avalanches form in the PPAC, which would be expected to give a correspondingly large increase in charge cloud size, relative to GEM. This in turn would provide a significant additional lowering of the charge density in the MSGC and a correspondingly higher gas gain.

To investigate this, we performed diffusion measurements with a PPAC as a preamplification structure. These measurements were performed in a similar way to the GEM measurements described in Section 3.1.1, and the results are presented in Fig. 4. One can see that the cloud spread is indeed larger than in the case of GEM. 
(a)
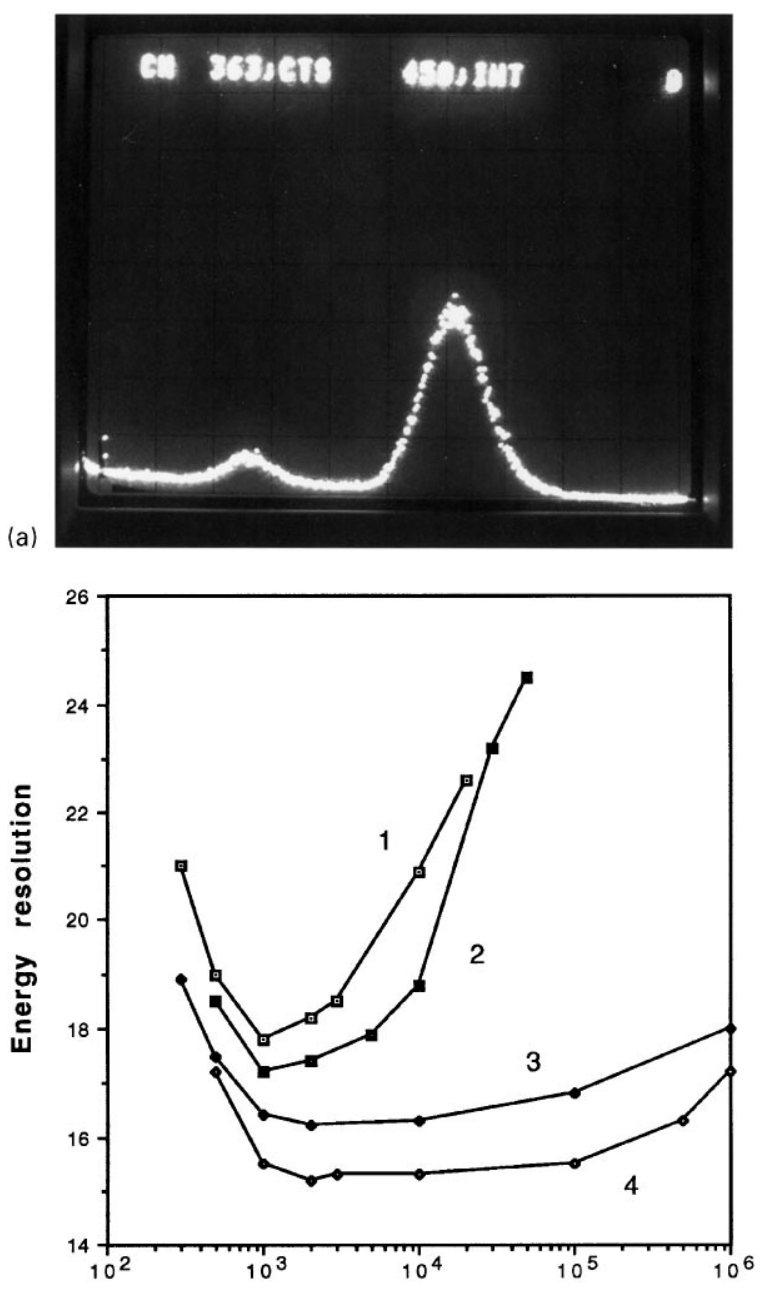

(b)

Gain

Fig. 7. Pulse-height spectrum from the MSGC \#1 with the PPAC (3 $\mathrm{mm}$ gap) as a preamplification structure. The distance between PPAC and MSGC was $10 \mathrm{~mm}$. (b) Energy resolution vs. total gain for MSGC \#1 $(1,2)$ and for MSGC \#1 with a PPAC (3 mm gap) as a preamplification structure $(3,4)$. For curves (1) and (3) the gas mixture is P10. For curves (2) and (4), the gas mixture is $\mathrm{Xe}+$ Isobutylene $(3 \%)$. Total pressure in all measurements was $1.05 \mathrm{~atm}$, and the input energy was $5.9 \mathrm{keV}$.

We also studied the breakdown limits of the preamplified MSGC in the presence of alpha particles. In these measurements the alphas were collimated perpendicular to the PPAC mesh and entered the drift region $5 \mathrm{~cm}$ above the PPAC. An effective "preamplification" of charge passed to the MSGC from the PPAC of $\sim 10$ could be achieved before sparking appeared in the PPAC. Reducing the drift space proportionally increased this gain as it reduced the amount of charge that enters.

Fig. 7 shows the resulting energy resolution of the MSGC coupled to the PPAC preamplification structure. Remarkably good resolution was obtained (15\% FWHM at $5.9 \mathrm{keV})$, even up to very high gains, and was approximately equal to that of the PPAC alone at the same total gain.

Finally, we also tested a PPAC with a smaller gap. The distinct advantage of this approach is its lower operating voltage, but unfortunately worse energy resolution $(\sim 19 \%$ at $6 \mathrm{keV})$ was obtained probably due to poorer control of geometrical inaccuracies. Its overall gain performance was the same as the larger gap device.

\section{Discussion}

Our previous study [14] showed the limitation in maximum achievable gains in microstrip-type detectors. In small pitch $(<1 \mathrm{~mm})$ detectors with substrates, this maximum is around $10^{4}$ whereas in designs without substrates, this maximum could reach $10^{5}$. In both cases (with and without substrates), limitations came from streamers which appear at some critical charge density in the avalanches. It is unfortunately quite difficult to restrict streamer development. They appear when the space charge of the avalanche starts to disturb the external electric field. In this case, photoelectrons produced by avalanche emission tend to collect in the remaining ion cloud, starting new avalanches, and this continuous process leads to streamer formation (see Ref. [3] for more details).

A way to significantly reduce this effect, as we have seen above, is by using preamplification structures to force diffusion of the primary charge cloud and permit higher gains at lower charge densities. However, as one can see from Fig. 2, in spite of this one cannot eventually avoid space charge effects and this puts an ultimate limit on the maximum collected charge of $2 \times 10^{5}$ in the GEM + MSGC configuration, after which a significant deviation from proportionality appears. This limit, $\sim 2 \times 10^{5}$, was typical for all mixtures tested at pressure of $1 \mathrm{~atm}$. In the case 
of the PPAC + MSGC, Fig. 6 shows that a higher overall gain was obtained $\sim 10^{6}$, and this was due to the fact that the PPAC structure gives higher diffusion than GEM.

One of the drawbacks of the PPAC as a preamplification structure is its previously mentioned low electron transparency. This problem is well known from the experience of exploring two-step PPAC amplification, where studies showed that the transparency is proportional to the ratio of the field outside to that inside the PPAC. In our case, the field ratio was $3 \%$ and the resulting transparency was on a similar level. It is well established that breakdown in PPACs occurs at a total charge of $10^{8}$ [15]. This gives a primary-charge-dependent limitation on the maximum achievable gain in the PPAC. For example, for $6 \mathrm{keV}$ photons, the maximum achievable gain will be $\sim 10^{6}$ and for alphas $\sim 10^{3}$. Since the transmission of our PPAC was a few \%, a maximum "preamplification" gain of the PPAC is around $10^{4}$ for $6 \mathrm{keV}$ and $\sim 10$ for alphas and other heavily ionizing particles.

It is known that PPACs can give very good energy resolutions, typically around $15 \%$ FWHM at $5.9 \mathrm{keV}$. Since at low MSGC gains the injected charge from the PPAC dominates the amplification statistics, the energy resolution of the PPAC + MSGC is similarly good (see Fig. 7).

It is interesting to compare the PPAC and GEM preamplification structures. Certainly GEM has a lot of advantages. It is a convenient and flexible structure which operates at very low voltages and has good transparency for electron clouds [5]. Conversely, the PPAC can give higher overall gains, provide superior energy resolution, operate at higher rates and is undamaged by sparks. Its drawbacks are the higher voltages needed for operation and the careful design necessary to ensure the parallelism of electrodes over the whole active area. As the transparency of the PPAC can be increased through higher field ratios, the high achievable overall gain is probably the most important feature of the PPAC + MSGC combination, since it then requires only one step of preamplification. In this heavily preamplified case, the MSGC may serve mainly for position measurements and may be replaced by any strip structure. Other preamplification structures may work equally well though, and our short-term plans include evaluation of MICROMEGAS [16,17].

Finally, we should note that the PPAC was used earlier as a successful preamplification structure for wire chambers [18,19]. Results obtained in Ref. [19] were very similar to ours with the MSGC: higher gains and much better energy resolutions were achieved compared with the bare MWPC.

\section{Conclusion}

Our previous study showed the fundamental limitations on maximum achievable gas gains in microstrip-type detectors. For conventional designs with substrates this is around $10^{4}$ while for designs without substrates, $10^{5}$ is possible. In this study, the addition of preamplifying structures was found to dramatically increase these values (up to $10^{6}$ ), to a point where space-charge non-linearities were the dominant limitations. An additional benefit of greatly enhanced energy resolution at high gains was found for the case where the microstrip gas counter was preamplified by a parallel plate avalanche chamber.

\section{Acknowledgements}

We thank F. Sauli for providing the GEM preamplification structure and for discussions. For discussions we also thank L. Ropelewski and R. Bellazzini.

\section{Appendix A}

If we denote by $n(\boldsymbol{P}, t)$ the density of free electrons in a gas then its evolution is given by the electrons transport and diffusion equation

$$
\frac{\partial n}{\partial t}=S-\nabla \cdot(n \boldsymbol{v})+D \nabla^{2} n,
$$

where $\boldsymbol{P}=(x, y, z)$ is the position vector, $\boldsymbol{v}(\boldsymbol{P})=\left(v_{x}, v_{y}, v_{z}\right)$ is the velocity field, $D$ is the electrons diffusion coefficient and $S$ is a source term 
given by

$S(\boldsymbol{P})=\alpha|\boldsymbol{v}| n$,

$\alpha$ being the first Townsend coefficient.

We are interested in the solution of this equation in the presence of a focusing or defocusing velocity field. To keep the problem simple we will consider a few physically reasonable simplifications that will allow us to derive an analytical solution:

(1) The velocity field is linearly approximated in the volume of interest,

$\boldsymbol{v}(\boldsymbol{P})=V_{0}+\boldsymbol{J P}$,

( $\boldsymbol{J}$ is the Jacobian matrix of $\boldsymbol{v}$ with respect to $\boldsymbol{P}$ ) and $\boldsymbol{J}$ is diagonal, taking $\boldsymbol{v}$ the form

$v_{u}(\boldsymbol{P})=V_{0 u}+J_{u u} u, \quad u=\{x, y, z\}$.

A material particle moving in this velocity field will follow the curve $\boldsymbol{P}_{0}(t)=\left(x_{0}, y_{0}, z_{0}\right)$, with

$u_{0}(t)=\left(u_{0}+\frac{V_{0 u}}{J_{u u}}\right) \exp \left(J_{u u} t\right)-\frac{V_{0 u}}{J_{u u}}, \quad u=\{x, y, z\}$.

(2) The source term is considered to be constant over the volume of interest and equal to its value at $\boldsymbol{P}_{0}$, becoming then a function of $t$ only.

$S=S(t)$

(3) The initial cloud is of a Gaussian shape with its center at position $\boldsymbol{P}_{0}(t=0)$.

Under the above simplifications Eq. (A.1) is separable and its solution is given by

$n(\boldsymbol{P}, t)=N_{0} s(t) g_{x}(x, t) g_{y}(y, t) g_{z}(z, t)$

with

$$
\begin{aligned}
& s(t)=\exp \left(\int_{0}^{t} S(\tau) \mathrm{d} \tau\right), \\
& g_{u}(u, t)=\frac{\exp \left(-\pi\left(u-u_{0}\right)^{2} / B_{u}\right)}{\sqrt{B_{u}}}, \quad u=\{x, y, z\}, \\
& B_{u}(t)=\left(B_{0 u} \frac{2 \pi D}{J_{u u}}\right) \exp \left(2 J_{u u} t\right)-\frac{2 \pi D}{J_{u u}}, \quad u=\{x, y, z\} .
\end{aligned}
$$

The usual Gaussian parameter $\sigma$ is given by $\sigma=\sqrt{B / 2 \pi}$ and $N_{0}$ is the initial number of electrons.

For Fig. 3 the following physical parameters were used:

$V_{0 x}=5 \mathrm{~cm} / \mu \mathrm{s} \quad V_{0 y}=V_{0 z}=0, \quad S=0 ;$

$J_{x x}=0, D=0.1 \mathrm{~m}^{2} / \mathrm{s}, J_{y y}=J_{z z}=0$ for Fig. 3(a) and $J_{y y}=J_{z z}= \pm 57 \mu \mathrm{s}^{-1}$ for Fig. 3(b),

corresponding to a constant (saturated) velocity along the $x$-axis and a focusing $\left(J_{y y}<0\right)$ or defocusing $\left(J_{y y}>0\right)$ effect along the $y$ - and $z$-axis. The linear diffusion parameter is $\sigma_{0}=200 \mu \mathrm{m} / \sqrt{\mathrm{cm}}$.

\section{References}

[1] V. Peskov et al., Nucl. Instr. and Meth. A 392 (1997) 89.

[2] V. Peskov, B.D. Ramsey, J. Kolodziejczak, P. Fonte, Nucl. Instr. and Meth. A 397 (1997) 243.

[3] P. Fonte, V.Peskov, B.D. Ramsey, Streamers in MSGC and other gaseous detectors, ICFA Instrum. Bulletin, 1997, http://www.slac.stanford.edu/pubs/icfa/.

[4] F. Sauli, Nucl. Instr and Meth. A 386 (1997) 531.

[5] R. Bouclier et al., Nucl. Instr. and Meth. A 396 (1997) 50.

[6] B.D. Ramsey et al., Nucl. Instr. and Meth. A 383 (1996) 424.

[7] N. Amos et al., Nucl. Instr. and Meth. A 384 (1997) 342.

[8] F. Sauli, Principles of operation of multiwire proportional and drift chambers, CERN 77-09, 1977.

[9] R. Bellazzini et al., Rivista del Nuovo Cimento, 17 (12) (1994), Editrice Compository, Bologna.

[10] E.D. Lozanski, Sov. Phys. Usp. 18 (11) (1976) 893.

[11] H. Raether, Electron avalanches and breakdowns in gases, Butterworths, Washington, 1964.

[12] G. Charpak et al., IEEE Trans. on Nucl. Sci. 35 (1) (1988) 483.

[13] P. Fonte, Ph.D. Thesis, Coimbra University, 1991.

[14] V. Peskov, B.D. Ramsey, P. Fonte, Breakdown features of various microstrip-type gas counters designs and their improvements, IEEE Trans. on Nucl. Sci. 45(3) (1998) 244.

[15] P. Fonte et al., Nucl. Instr. and Meth. A 305 (1991) 91.

[16] I. Giomataris et al., preprint DAPNIA/SED 95-04, 1995.

[17] B.D. Ramsey et al., Instrumentation for X-ray astronomy from high-altitude balloons: recent developments and future plans, NASA/MSFC preprint, 1998.

[18] A. Breskin et al., Nucl. Instr. and Meth. 161 (1979) 19.

[19] B.D. Ramsey et al., Nucl. Instr. and Meth. A 248 (1986) 550 . 